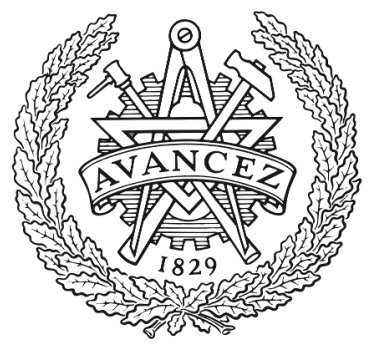

CHALMERS

UNIVERSITY OF TECHNOLOGY

\title{
Highly Permeable Fluorinated Polymer Nanocomposites for Plasmonic Hydrogen Sensing
}

Downloaded from: https://research.chalmers.se, 2023-04-26 07:49 UTC

Citation for the original published paper (version of record):

Östergren, I., Pourrahimi, A., Darmadi, I. et al (2021). Highly Permeable Fluorinated Polymer

Nanocomposites for Plasmonic Hydrogen Sensing. ACS Applied Materials \& Interfaces, 13(18):

21724-21732. http://dx.doi.org/10.1021/acsami.1c01968

N.B. When citing this work, cite the original published paper. 


\title{
Highly Permeable Fluorinated Polymer Nanocomposites for Plasmonic Hydrogen Sensing
}

\author{
Ida Östergren, ${ }^{\text {II }}$ Amir Masoud Pourrahimi, ${ }^{\text {II }}$ Iwan Darmadi, ${ }^{\text {II }}$ Robson da Silva, Alicja Stolaś, Sarah Lerch, \\ Barbara Berke, Manuel Guizar-Sicairos, Marianne Liebi, Giacomo Foli, Vincenzo Palermo, \\ Matteo Minelli, Kasper Moth-Poulsen,* Christoph Langhammer,* and Christian Müller*
}

Cite This: ACS Appl. Mater. Interfaces 2021, 13, 21724-21732

Read Online

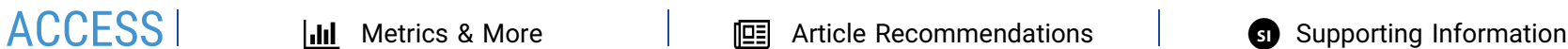

ABSTRACT: Hydrogen $\left(\mathrm{H}_{2}\right)$ sensors that can be produced en masse with cost-effective manufacturing tools are critical for enabling safety in the emerging hydrogen economy. The use of melt-processed nanocomposites in this context would allow the combination of the advantages of plasmonic hydrogen detection with polymer technology; an approach which is held back by the slow diffusion of $\mathrm{H}_{2}$ through the polymer matrix. Here, we show that the use of an amorphous fluorinated polymer, compounded with colloidal Pd nanoparticles prepared by highly scalable continuous flow synthesis, results in nanocomposites that display a high $\mathrm{H}_{2}$ diffusion coefficient in the order of $10^{-5} \mathrm{~cm}^{2} \mathrm{~s}^{-1}$. As a result, plasmonic optical hydrogen detection with melt-pressed

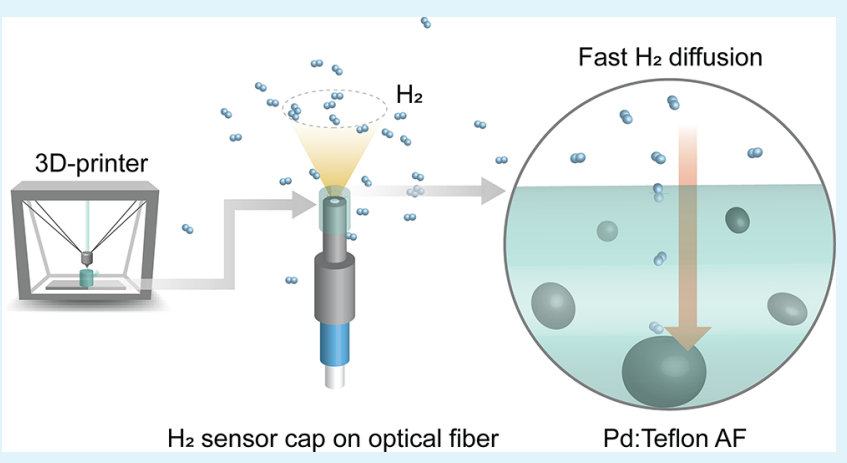
fluorinated polymer nanocomposites is no longer limited by the diffusion of the $\mathrm{H}_{2}$ analyte to the Pd nanoparticle transducer elements, despite a thickness of up to $100 \mu \mathrm{m}$, thereby enabling response times as short as $2.5 \mathrm{~s}$ at $100 \mathrm{mbar}\left(\equiv 10\right.$ vol. \%) $\mathrm{H}_{2}$. Evidently, plasmonic sensors with a fast response time can be fabricated with thick, melt-processed nanocomposites, which paves the way for a new generation of robust $\mathrm{H}_{2}$ sensors.

KEYWORDS: fluorinated polymer, palladium nanoparticle, melt-processed nanocomposite, hydrogen permeability and diffusion, plasmonic sensing

\section{INTRODUCTION}

Hydrogen gas $\left(\mathrm{H}_{2}\right)$ is an efficient carbon-free energy carrier that can be extracted from sources that include natural gas, ${ }^{1}$ biomass, ${ }^{2}$ or water/wastewater. ${ }^{3,4}$ In contrast to the combustion of fossil fuels, the conversion of $\mathrm{H}_{2}$ to electrical energy in a fuel cell produces only water, without any $\mathrm{CO}_{2}$ emissions. ${ }^{5}$ Therefore, a transition from carbon-based fuels to $\mathrm{H}_{2}$ could ultimately play a key role in mitigating climate change. ${ }^{6}$ However, $\mathrm{H}_{2}$ gas is odorless, colorless, and highly flammable under ambient conditions when mixed with air, which causes considerable safety concerns. The ability to rapidly detect even minute amounts of $\mathrm{H}_{2}$ gas is therefore of paramount importance at all stages of the hydrogen energy cycle from production to transport, storage, and consumption. As a result, novel sensor technologies that can be used for onthe-spot detection of $\mathrm{H}_{2}$ leaks are currently in high demand.

A $\mathrm{H}_{2}$ sensor must be able to detect any leaks within a few seconds, depending on the specific application. ${ }^{8}$ Among different types of detection principles, optical sensing based on localized surface plasmon resonance (LSPR) is particularly promising because it offers a unique combination of high selectivity and accuracy, fast response, and spark-free operation due to remote readout. ${ }^{8}$ In this context, $\mathrm{Pd}$ is the most commonly used element and model system because it readily sorbs hydrogen without a sizable energy barrier and undergoes a reversible phase transformation from metal to metal hydride at room temperature, which gives rise to a sizable optical contrast. ${ }^{9}$ State-of-the-art $\mathrm{H}_{2}$ plasmonic sensors therefore comprise either pure $\mathrm{Pd}$ or $\mathrm{Pd}$ alloy nanoparticles ${ }^{10}$ that are shaped directly on a surface using nanolithography, ${ }^{8,11}$ are grown $^{12,13}$ or deposited onto a surface using vacuum deposition, ${ }^{14,15}$ or are prepared by colloidal synthesis. ${ }^{16}$ Despite considerable advances in terms of both sensor fabrication and performance, the large-scale implementation of $\mathrm{H}_{2}$ plasmonic sensors is still lacking, partly due to cost and scalability issues with the nanofabrication methods used to produce two-dimensional (2D) arrays of hydrogen-sensitive plasmonic nanoparticles on flat surfaces. As a result, the

Received: January 29, 2021

Accepted: April 15, 2021

Published: April 28, 2021 


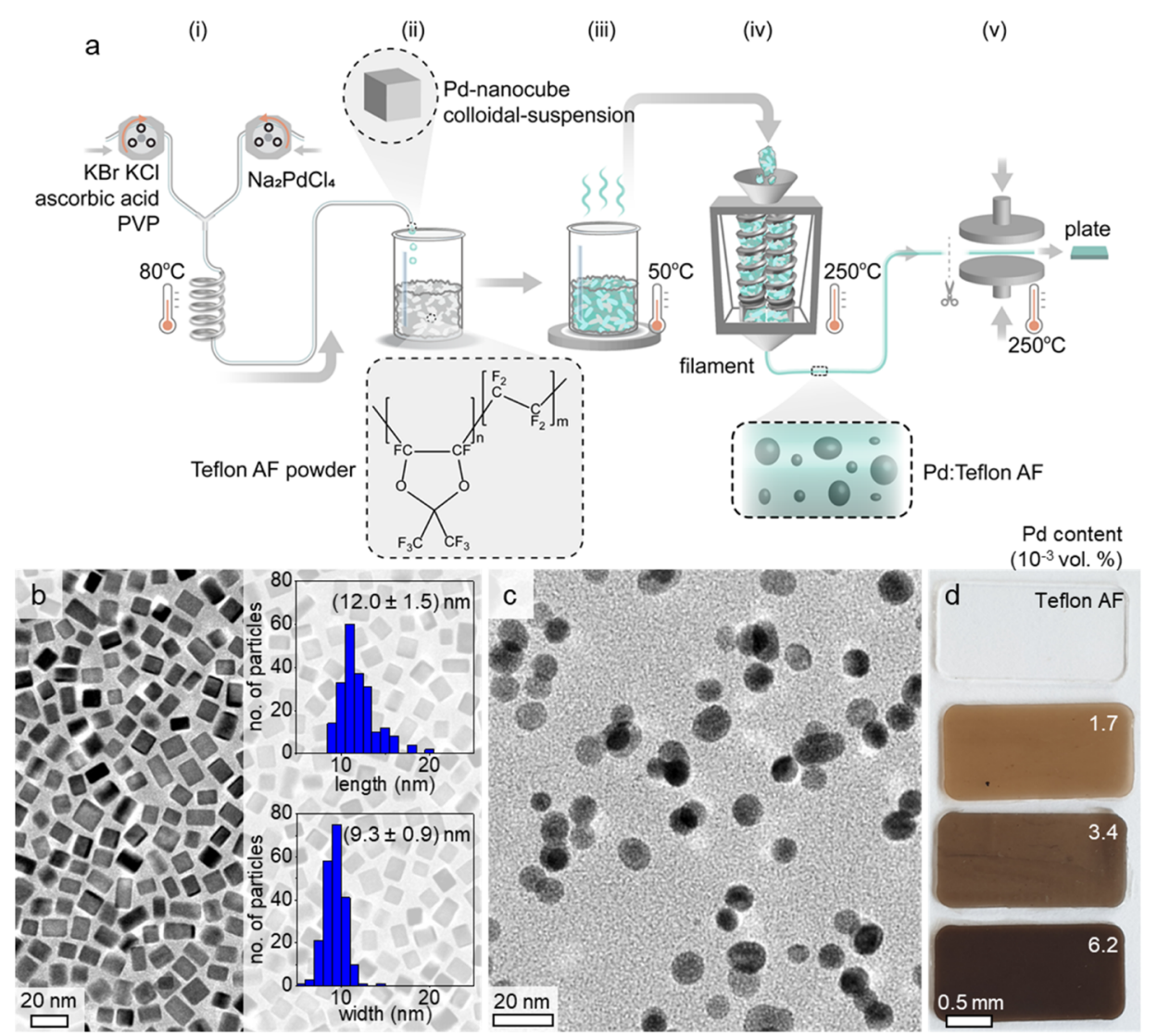

Figure 1. Polymer nanocomposite preparation. (a) Material preparation work flow entailing (i) flow synthesis of PVP-capped Pd nanocubes, (ii) mixing of the Pd nanocube suspension (isopropanol medium) with the polymer, Teflon AF, (iii) drying of the Pd nanoparticle:polymer mixture, (iv) melt extrusion, and (v) melt pressing; (b) TEM image of Pd nanocubes with size (rectangle with length and width) histograms; (c) TEM image of Pd nanoparticles in Teflon AF; and (d) melt-pressed $0.5 \mathrm{~mm}$-thick plates of neat Teflon AF and its nanocomposites.

attention is increasingly shifting to more cost-effective optical sensors that can be realized with scalable production methods. ${ }^{17,18}$ Therefore, we argue that it would be particularly desirable to harness the wealth of readily available polymer nanocomposite processing methods, such as solution processing, ${ }^{19,20}$ sputter deposition, ${ }^{21-23}$ and especially bulk processing methods, such as melt compounding. ${ }^{16,24}$

To this end, we have recently shown that plasmonic $\mathrm{H}_{2}$ sensors can be fabricated with three-dimensional (3D) geometries. ${ }^{16}$ Specifically, we prepared polymer nanocomposites composed of $\mathrm{Pd}$ nanoparticles, initially obtained by colloidal synthesis, and poly(methyl methacrylate) (PMMA), which we were able to shape using polymer processing methods such as extrusion, molding, and fused filament fabrication (FFF), a common 3D printing technique. While these Pd:PMMA nanocomposites could be used to fabricate fully functional $\mathrm{H}_{2}$ sensors with excellent long-term stability, the sensor elements suffered from a slow response and recovery time because of the slow $\mathrm{H}_{2}$ gas diffusion through the polymer matrix. $^{16}$

Here, we demonstrate that the judicious selection of a polymer with a high permeability for $\mathrm{H}_{2}$ enables a fast response time as short as $2.5 \mathrm{~s}$ when exposing $100 \mu \mathrm{m}$-thick samples to a $100 \mathrm{mbar}_{2} \mathrm{H}_{2}$ pressure step from vacuum. This remarkable performance was enabled by the use of the amorphous fluorinated polymer poly(4,5-difluoro-2,2-bis(trifluoromethyl)1,3-dioxole-co-tetrafluoroethylene) (Teflon $\mathrm{AF}$ ) as the matrix material, which displays a substantially higher $\mathrm{H}_{2}$ permeability due to a considerably larger fractional free volume. ${ }^{25,26}$ In addition, the sensor shows astonishing sensitivity with an extremely low limit of detection (LOD) of $0.03 \mathrm{mbar}$ ( $\equiv 0.003$ vol. $\% \equiv 30 \mathrm{ppm}$ ), which is among the best optical hydrogen sensors reported. ${ }^{10}$ We compounded Teflon AF with colloidal Pd nanoparticles, produced by a scalable continuous flow synthesis process, ${ }^{27}$ to create a highly hydrogen-permeable and hydrogen-sensitive nanocomposite material. Finally, we 3Dprinted a fully functional sensor cap, which could be placed on a fiber optic connector and facilitated stable hydrogen sensing. The sensor exhibits outstanding stability under the 100 cycle (equivalent to $18 \mathrm{~h}$ ) sensing test in a synthetic air background.

\section{RESULTS AND DISCUSSION}

To prepare well-defined Pd nanoparticles in terms of size and shape, we used a continuous flow synthesis method, ${ }^{27}$ which provided single-crystal Pd nanoparticles coated/stabilized with poly(vinylpyrrolidinone) (PVP) (Figure 1a). Transmission electron microscopy (TEM) revealed that the obtained $\mathrm{Pd}$ single-crystal nanoparticles (enclosed by low-index ${ }^{28}$ facets with an edge truncation of $15 \%$; Figure S1) had a uniform cubic shape with a narrow size distribution (Figure $1 \mathrm{~b}$ ). With a zeta potential of $-35 \mathrm{mV}$ at $\mathrm{pH} 5.6$, the $\mathrm{Pd}$ nanocubes displayed a high degree of colloidal stability in the water-based reaction medium. To improve the compatibility with hydrophobic polymers such as Teflon AF targeted here, we carried out medium exchange by precipitation of the as-obtained $\mathrm{Pd}$ nanocubes from aqueous suspension with acetone, followed by redispersion in isopropanol. 
The nanocomposite material was prepared by mixing the suspension of the $\mathrm{Pd}$ nanocubes with polymer powder, followed by evaporation of isopropanol at 40 to $50{ }^{\circ} \mathrm{C}$ overnight, resulting in polymer powder slurry with welldistributed Pd nanocubes. Finally, the material was molten in a microcompounder and extruded into filaments that could be used for melt pressing of thin plates, ranging from 50 to 650 $\mu \mathrm{m}$ in thickness (Figure 1a), or FFF-type 3D printing of more complex objects (will be discussed later). TEM images of cryofractured melt-pressed plates confirmed the presence of solitary $\mathrm{Pd}$ nanoparticles without aggregation (Figures 1c and S2). We note, however, that the Pd nanoparticles now had a more spherical shape, as compared to the nanocubes with sharp edges obtained from the synthesis (cf. Figure $1 \mathrm{~b}$ ). This change in shape was observed across entire samples (see Figure S2 for additional images). We attribute the restructuring of nanoparticles to the relatively high processing temperature of 250 ${ }^{\circ} \mathrm{C}$ (see Figure 1a), similar to the reconstruction of bare $\mathrm{Pd}$ nanoparticles at 150 to $220{ }^{\circ} \mathrm{C}$ reported by Pekkari et al. ${ }^{27}$ and not observed in our previous paper where the nanocomposite was processed at a lower temperature of $200{ }^{\circ} \mathrm{C}$. ${ }^{16}$ The $\mathrm{Pd}$ content in the melt-pressed plates scaled with the amount of Pd present during the initial synthesis step (Figure S3a), which indicates that no material is lost during the various processing steps. Accordingly, the appearance of melt-pressed plates ranged from completely transparent in the case of neat Teflon $\mathrm{AF}$ to dark brown in the case of a nanocomposite containing $6.2 \times 10^{-3}$ vol. \% Pd (Figure 1d), due to the LSPR of the Pd nanoparticles. ${ }^{29}$ The LSPR frequency is independent of the Pd concentration (Figure S3b). The difference in appearance is due to higher light absorption, which increases with nanoparticle concentration (Beer-Lambert law ${ }^{30,31}$ ).

To assess the degree of dispersion of the Pd nanoparticles in melt-pressed Pd:Teflon AF nanocomposites at a more global scale, we carried out scanning small-angle X-ray scattering (SAXS) measurements (Figures 2 and S4). For this purpose,
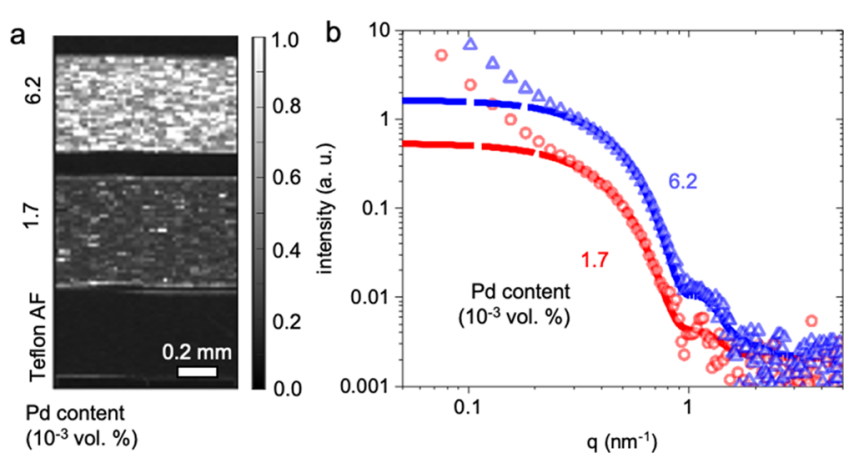

Figure 2. SAXS of Pd:Teflon AF nanocomposites. (a) Scanning-SAXS images of melt-pressed and (b) background-corrected SAXS scattering curves of Pd:Teflon AF nanocomposites (open symbols) with the corresponding ellipsoid fit (solid line); the scattering intensity of neat Teflon AF has been subtracted. The deviation from the fitted curve in the low $q$ area is caused by the interactions between the nanoparticles and the Teflon AF matrix.

we prepared cross sections of melt-pressed plates containing 1.7 and $6.2 \times 10^{-3}$ vol. $\%$ Pd. Using a $25 \times 10 \mu \mathrm{m}$ step size, we recorded scattering patterns over the chosen sample area of $\sim 0.5 \times 1 \mathrm{~mm}^{2}$. The corresponding scanning-SAXS images (Figure $2 \mathrm{a}$ ) show the average scattering intensity analyzed in a $q$-range of 0.2 to $0.69 \mathrm{~nm}^{-1}$. The scattering intensity appears similar at each scan position, which suggests that the $\mathrm{Pd}$ nanoparticles are homogeneously distributed in the Teflon AF matrix (Figure 2a; note that bright spots arise due to small local concentration differences). No preferential orientation was observed in the Pd:Teflon AF composites (Figure S4). Quantitative analysis of SAXS scattering curves revealed that the dispersed $\mathrm{Pd}$ nanoparticles had an average diameter between 8 and $11 \mathrm{~nm}$ obtained from ellipsoid fitting (Figure $2 b$ ), which is in agreement with our TEM analysis (cf. Figure 1c) and confirms that the majority of nanoparticles have undergone restructuring into ellipsoids and are well dispersed, that is, no or only very few aggregates are present in the nanocomposite.

To determine the permeability of $\mathrm{H}_{2}$ through melt-pressed plates of neat Teflon AF and of nanocomposites containing the Pd nanoparticles, we used a custom-made setup that allows measuring of the time lag of gas transport through polymer films, which separate the feed and the initially empty permeate compartment. ${ }^{32}$ The feed side of the sample is exposed to $\mathrm{H}_{2}$, which absorbs and diffuses through the plate and then desorbs on the permeate side, leading to a gradual increase in pressure until the steady-state conditions are obtained (linear increase with time of permeated $\mathrm{H}_{2}$ molecules, i.e., constant flux). The time lag at the beginning of the measurement and the slope in the steady-state region allow us to determine the diffusion coefficient $D$ and the permeability $P$, respectively (Figure 3 ). To benchmark the high permeability of Teflon AF, we also included semicrystalline poly(vinylidene fluoride) (PVDF) and amorphous PMMA in our study. For neat Teflon AF, we obtain $D \approx 2.3 \times 10^{-5} \mathrm{~cm}^{2} \mathrm{~s}^{-1}$ and $P \approx 745$ barrer, which are both considerably higher than values obtained for PMMA or PVDF (Table 1), indicating that Teflon AF is a superior choice for the realization of nanocomposites that are to be used for rapid $\mathrm{H}_{2}$ sensing. We observe that the presence of $\mathrm{Pd}$ nanoparticles in the Teflon AF matrix does not affect $P$ and only slightly reduces $D$ (Table 1 ), which is in agreement with studies of $\mathrm{Pd}$ in other polymers. ${ }^{19}$ The higher diffusion coefficient of Teflon AF compared to PMMA, which are both amorphous [cf. differential scanning calorimetry (DSC) thermograms in Figure S5] can be rationalized by considering the fractional free volume (FFV) since $D \propto \mathrm{e}^{-B / \mathrm{FF}},{ }^{28}$ where $B$ is a constant. Teflon AF tends to pack poorly in the glassy state, ${ }^{26,33}$ which gives rise to a higher FFV and hence $D$ than PMMA. Instead, the lower diffusion coefficient of PVDF can be ascribed to the semicrystalline nature of the polymer (Figure S5).

As the next step, we evaluated the effectiveness of Pd:Teflon AF nanocomposites for plasmonic optical $\mathrm{H}_{2}$ sensing by spectrally resolved monitoring of the optical extinction of a melt-pressed plate during $\mathrm{H}_{2}$ exposure at different pressures (Figure 4a). The spectra feature distinct changes in the selfreferenced extinction, $\varepsilon$, upon exposure to $\mathrm{H}_{2}$, with a minimum and maximum emerging at $\lambda_{\min } \approx 480 \mathrm{~nm}$ and at $\lambda_{\max } \approx 975$ $\mathrm{nm}$, respectively (Figure $4 \mathrm{~b}$ ). Here, self-referenced extinction means that the measured extinction signal is normalized with respect to the first spectrum measured for the composite in the nonhydrogenated state. We chose to use the difference in extinction $\Delta \varepsilon=\varepsilon\left(\lambda_{\max }\right)-\varepsilon\left(\lambda_{\min }\right)$ as the descriptor for the optical response to hydrogen to cancel the spectrum baseline drift. Using this descriptor, we then constructed a pressurecomposition isotherm (Figure $4 \mathrm{~b}$ ), which reproduces the characteristics of the palladium-hydrogen system, with the solid solution $\alpha$-phase present at low $\mathrm{H}_{2}$ pressure, the $\beta$-phase 

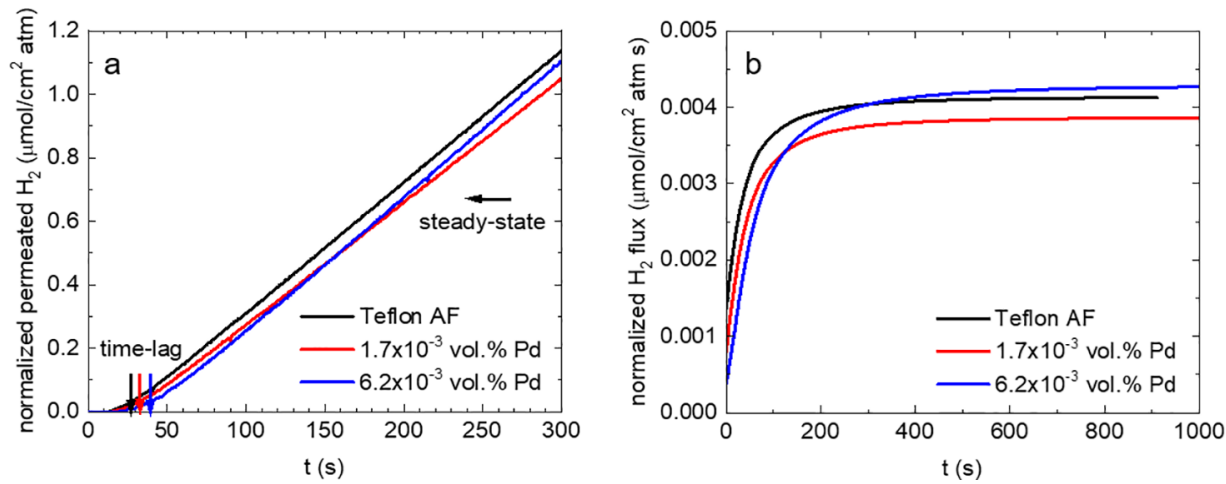

Figure 3. $\mathrm{H}_{2}$ permeability measurement based on the time-lag method. (a) Normalized permeated $\mathrm{H}_{2}$ and (b) normalized flux of $\mathrm{H}_{2}$ per unit area through $600 \mu \mathrm{m} \pm 25 \mu \mathrm{m}$-thick melt-pressed plates of neat Teflon AF and Pd:Teflon AF nanocomposites (the flux was normalized with respect to the sample thickness).

Table 1. $\mathrm{H}_{2}$ Permeability $P$ and Diffusivity $D$ from the Time-Lag Method, Calculated after a Long Desorption Time (Overnight) and Characteristic Diffusion Time CDT $=d^{2} / 6 D$ of Plates with Thickness $d$

\begin{tabular}{|c|c|c|c|c|}
\hline material & $d(\mu \mathrm{m})$ & $\boldsymbol{P}$ (barrer) & $D\left(\mathrm{~cm}^{2} / \mathrm{s}\right)$ & CDT (s) \\
\hline PMMA & $262 \pm 9$ & $4.2 \pm 0.2$ & $(6.6 \pm 0.4) \times 10^{-7}$ & $177 \pm 24$ \\
\hline Teflon AF & $589 \pm 16$ & $745 \pm 2$ & $(2.32 \pm 0.04) \times 10^{-5}$ & $25 \pm 1$ \\
\hline$+1.7 \times 10^{-3}$ vol. $\% \mathrm{Pd}^{a}$ & $586 \pm 9$ & $706 \pm 2$ & $(1.9 \pm 0.1) \times 10^{-5}$ & $30 \pm 1$ \\
\hline$+6.2 \times 10^{-3}$ vol. $\% \mathrm{Pd}^{a}$ & $608 \pm 16$ & $799 \pm 5$ & $(1.5 \pm 0.2) \times 10^{-5}$ & $41 \pm 4$ \\
\hline PVDF & $250 \pm 38$ & $0.48 \pm 0.02$ & $(5.9 \pm 0.2) \times 10^{-8}$ & $1780 \pm 55$ \\
\hline
\end{tabular}

${ }^{a}$ For Teflon AF nanocomposites, $D$ represents an effective value that also accounts for the hydride formation reaction. 1 barrer $=10^{-10} \mathrm{~cm}^{3}$ $(\mathrm{STP}) \mathrm{cm} /\left(\mathrm{cm}^{2} \mathrm{~s} \mathrm{cmHg}\right)$.

present at high $\mathrm{H}_{2}$ pressure, and an equilibrium plateau where both $\alpha$ - and $\beta$-phase coexist during the first-order phase transformation. ${ }^{9}$ We also note that hysteresis occurs during pressure cycling, showing that the hydride decomposition occurs at a lower pressure as compared to palladium-hydride formation, as a consequence of lattice strain and the corresponding energy barriers due to the presence of hydrogen in the host lattice. ${ }^{9}$ The characteristic shape of the isotherm also confirms that the observed changes in extinction upon $\mathrm{H}_{2}$ exposure arise due to absorption/desorption of hydrogen by the Pd nanoparticles, as anticipated. In addition, a polymer coating is known to shift the Pd plateau pressure due to induced strain. ${ }^{8}$ Therefore, the plateau pressure shown in Figure $4 \mathrm{~b}$ is likely to be distinct from that of Pd without Teflon AF. Furthermore, the pressure-composition isotherms recorded for different Pd concentrations (Figure S6) do not indicate a correlation between the plateau pressures and the $\mathrm{Pd}$ concentration. We explain the variation of the plateau pressure with batch-to-batch differences of the nanoparticles, not only in shape and size but also in the internal nanostructure. ${ }^{34,35}$

Now turning our analysis and discussion to the targeted application of the nanocomposite as optical plasmonic hydrogen sensors, we focus on the assessment of the response and recovery times, since they are vital aspects of a $\mathrm{H}_{2}$ sensor. Therefore, we investigated the $\mathrm{H}_{2}$ absorption and desorption kinetics of 50 to $650 \mu \mathrm{m}$-thick plates, by monitoring $\Delta \varepsilon$ upon a stepwise increase in $\mathrm{H}_{2}$ pressure from 0 to $100 \mathrm{mbar}$ (Figures $4 \mathrm{c}$ and $\mathrm{S} 7$ ). We define the response time $t_{50}$ as the time it takes to reach $50 \%$ of the total signal of the new steady state (cf. Figure $4 c$ ) and observe that $t_{50}$ scales quadratically with the plate thickness, $d$ (Figure $4 \mathrm{~d}$ ), indicating that the response is controlled by the diffusion of $\mathrm{H}_{2}$ through the polymer matrix. Applying our previously established $\operatorname{model}^{16}$ for the diffusionlimited $\mathrm{H}_{2}$ sorption kinetics of nanocomposites to this data set, we obtain a diffusion coefficient of $D \approx 1.6 \times 10^{-5} \mathrm{~cm}^{2} \mathrm{~s}^{-1}$, which is in excellent agreement with values obtained from direct gas permeation measurements (Table 1). A similar analysis of the desorption kinetics likewise shows a quadratic dependence of $t_{50} \propto d^{2}$ and yields a comparable value of $D \approx$ $3.1 \times 10^{-5} \mathrm{~cm}^{2} \mathrm{~s}^{-1}$ (Figure S8). We also studied the impact of Pd concentration on $t_{50}$ using $100 \mu$ m-thick plates (Figure S9). Both absorption and desorption $t_{50}$ do not depend on the concentration of Pd nanoparticles (Figure S10). This can be understood from two perspectives. First, the characteristic diffusion time (CDT $\left.=d^{2} / 6 D\right)$ of hydrogen through a thickness of $100 \mu \mathrm{m}$ is about $0.8 \mathrm{~s}$ (cf. Table 1 ), which is less than the typical $\mathrm{H}_{2}$ absorption time of a few seconds, reported for similar $\mathrm{Pd}$ nanoparticles; hence, the $\mathrm{H}_{2}$ concentration gradient through the plate is negligible. ${ }^{16}$ Second, the investigated $\mathrm{Pd}$ concentration range is much lower than the amount of the hydrogen dissolved in the polymer matrix. Consequently, these two factors cause the apparent hydrogenation time to only depend on the hydrogen uptake rate per nanoparticle. ${ }^{16}$

The optical contrast $\mathrm{OC}=\Delta \varepsilon_{\mathrm{PdHx}}-\Delta \varepsilon_{\mathrm{Pd}}$, which directly determines the sensitivity and signal-to-noise ratio of a sensor, is here defined as the difference in extinction $\Delta \varepsilon_{\mathrm{PdHx}}$ when $\mathrm{Pd}$ is fully hydrogenated at a pressure of $100 \mathrm{mbar}_{2}$ minus the difference in extinction $\Delta \varepsilon_{\mathrm{Pd}}$ of the nonhydrogenated state. We find that the optical contrast increases linearly with plate thickness at constant $\mathrm{Pd}$ concentration, that is, OC $\propto d$ (Figure 4e). A similar trend can also be achieved by increasing the $\mathrm{Pd}$ nanoparticle loading, in agreement with the Beer-Lambert law. ${ }^{30,31}$ These two strategies to enhance the optical contrast will be relevant for operation at elevated temperatures, for example, in connection to a fuel cell because the sensitivity of Pd-hydride based sensors tends to decrease due to lower hydrogen solubility. ${ }^{36}$ 


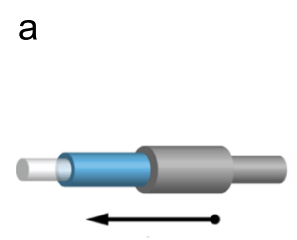

to spectrophotometer

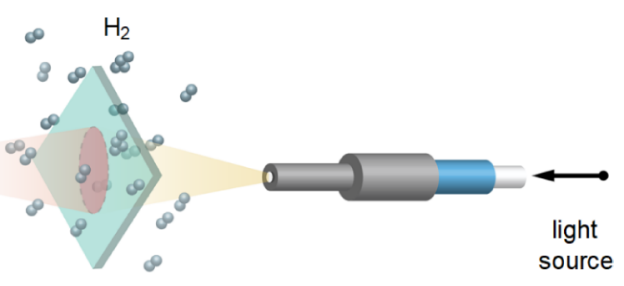

Pd:Teflon AF plate

source

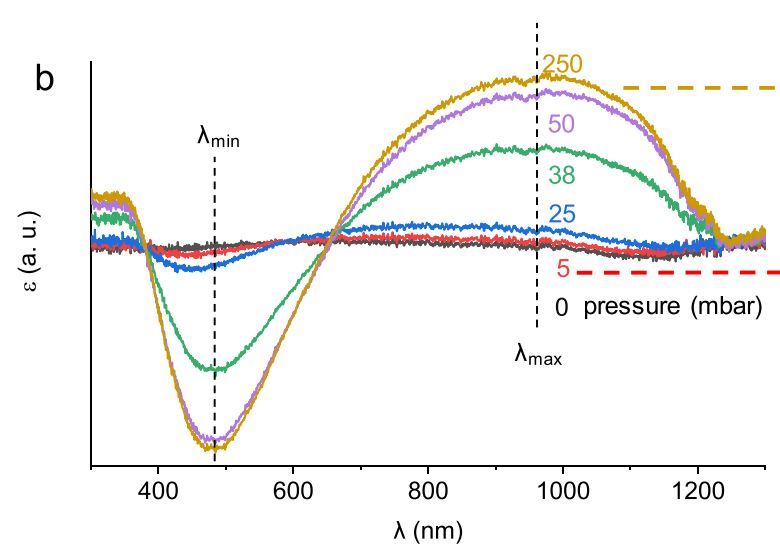

C

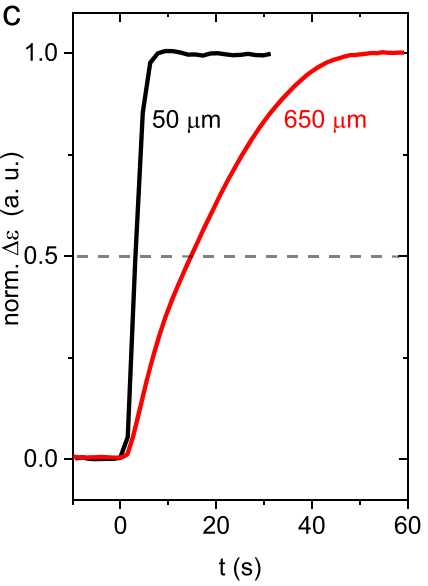

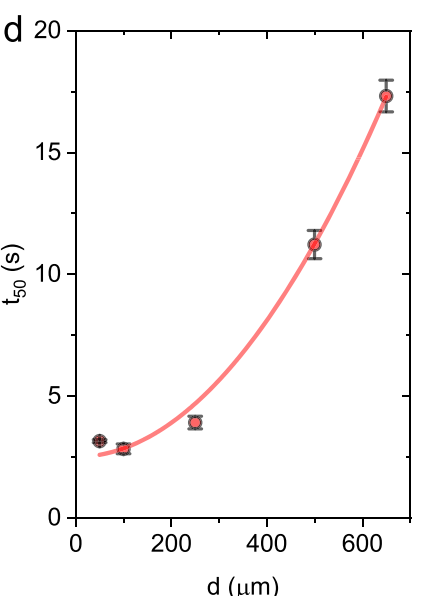

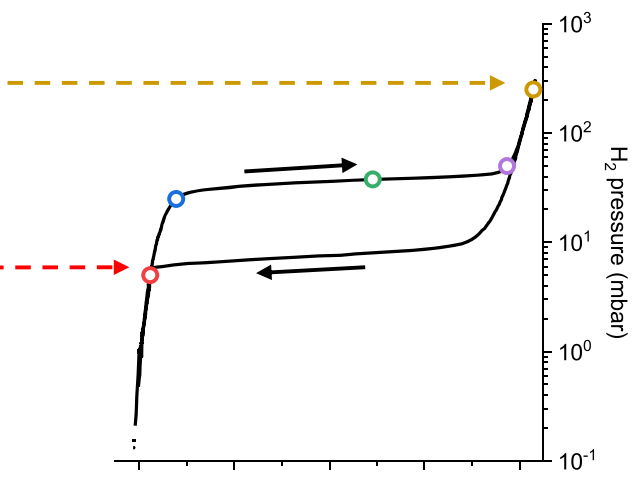

$\Delta \varepsilon$ (a. u.)

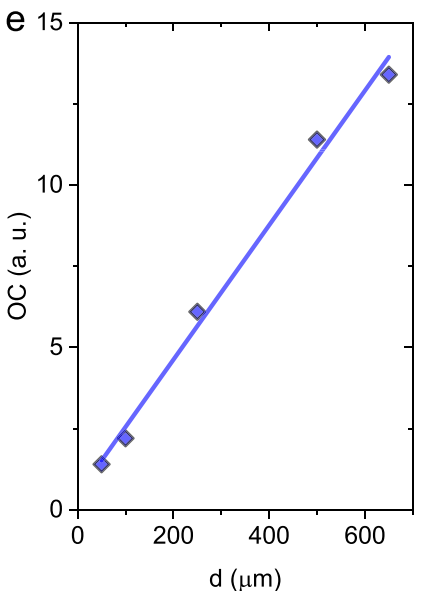

Figure 4. Plasmonic $\mathrm{H}_{2}$ sensing. (a) Schematic of optical $\mathrm{H}_{2}$ detection via extinction measurements through a melt-pressed nanocomposite plate; (b) selected self-referenced extinction spectra of a $100 \mu$ m-thick melt-pressed plate composed of Pd:Teflon $\mathrm{AF}\left(12.5 \times 10^{-3}\right.$ vol. \% Pd) during exposure to increasing $0-250 \mathrm{mbar} \mathrm{H}_{2}$ pressure at $30{ }^{\circ} \mathrm{C}$ together with their pressure-composition isotherm constructed using the difference in extinction $\Delta \varepsilon=\varepsilon\left(\lambda_{\max }\right)-\varepsilon\left(\lambda_{\min }\right)$ as the readout descriptor. A similar isotherm construction is also shown for decreasing hydrogen pressure from 250-0 mbar $\mathrm{H}_{2}$. Here, self-referenced extinction corresponds to the use of an extinction spectrum measured from a plate in the nonhydrogenated state as the optical reference. (c) Normalized $\Delta \varepsilon$ of 50 and $650 \mu$ m-thick melt-pressed plates of Pd:Teflon AF (3.4 $\times 10^{-3}$ vol. \% Pd) upon a stepwise increase in $\mathrm{H}_{2}$ pressure from 0 to $100 \mathrm{mbar}_{2}$ (the $\mathrm{H}_{2}$ valve opens at $t=0$ ); $(\mathrm{d}) \mathrm{H}_{2}$ absorption time or "response time" of the plate, $t_{50}$, defined as the time it takes to reach $50 \%$ of the total signal change to the new steady state versus the plate thickness $d$ (error bars are the standard deviation of five identical measurements). Also plotted is a quadratic fit $t_{50}=3.5 \times 10^{-5} \mathrm{~s} \mu \mathrm{m}^{-2} d^{2}+2.5 \mathrm{~s}$ (red line) and (e) optical contrast (OC) (see the main text for definition) as a function of plate thickness $d$, plotted together with a linear fit $\mathrm{OC}=0.02 \mu \mathrm{m}^{-1} d+0.48$ (blue line).

However, while a thick plate results in a high signal-to-noise ratio, it also increases the response time of the sensor due to longer diffusion paths. Therefore, we argue that a thickness of $100 \mu \mathrm{m}$ is the optimal choice with a $t_{50} \approx 2.5 \mathrm{~s}$ (pressure step of 0 to $100 \mathrm{mbar}$ ), since it does not decrease considerably for thinner samples (cf. Figure 4d). The optical contrast can instead be adjusted by selecting a sufficiently high $\mathrm{Pd}$ nanoparticle concentration, which does not influence $t_{50}$ (Figure S10). We note that the response time of the sensor with optimal thickness is higher compared to state-of-the-art hydrogen sensors. ${ }^{10}$ However, since in our case, the response time is limited by the hydrogen absorption kinetics of the Pd nanoparticles, in the next generation versions of sensors, it can be further improved by, for example, using a PdAu alloy, ${ }^{37} \mathrm{Pd}$ nanoparticles with specific facets/vertices, ${ }^{38}$ or a nonhalidebased nanoparticle stabilizer. ${ }^{39}$

To examine the LOD of the sensor, we tested the response of 50 and $500 \mu \mathrm{m}$-thick samples to low hydrogen pressures in the $\alpha$-phase regime of the Pd (Figure S11). We define the LOD as equal to three times the noise level ( $\mathrm{LOD}=3 \sigma=0.03$ a.u.). The 50 and $500 \mu \mathrm{m}$ samples feature LODs of $0.84 \mathrm{mbar}$ ( $\equiv 0.084$ vol. $\% \equiv 840 \mathrm{ppm}$ under ambient conditions) and 0.03 mbar ( $\equiv 0.003$ vol. $\% \equiv 30 \mathrm{ppm}$ ), respectively. To put these values into perspective, we note that both these LODs are below the limit required by some national agencies ( 0.1 vol. $\%) .{ }^{10}$ Furthermore, our results indicate that one strategy to 
amplify the optical response (i.e., the LOD) is to further increase the plate thickness.

Furthermore, it is worth discussing that the sensor sensitivity can be further improved by increasing the nanoparticle size. The nanoparticles we used in this study are very small and their LSPR wavelength is in the UV spectral range (Figure $\mathrm{S} 3 \mathrm{~b})$. At the same time, the sensitivity of plasmonic Pd-based sensors is proportional to the LSPR wavelength of the particles in the nonhydrogenated state, which, in turn, is proportional to the nanoparticle size. ${ }^{40,41}$ In this study, we chose to work with $\mathrm{Pd}$ nanoparticles that are $10 \mathrm{~nm}$ in size because the synthesis time increases for larger nanoparticles.

In a last set of experiments, we used FFF to 3D-print sensor caps with a bottom wall thickness of $200 \mu \mathrm{m}$. The cap was fit by "plug and play" onto an SMA 905 fiber optic connector (Figure 5a,b), which was inserted into a custom-built test
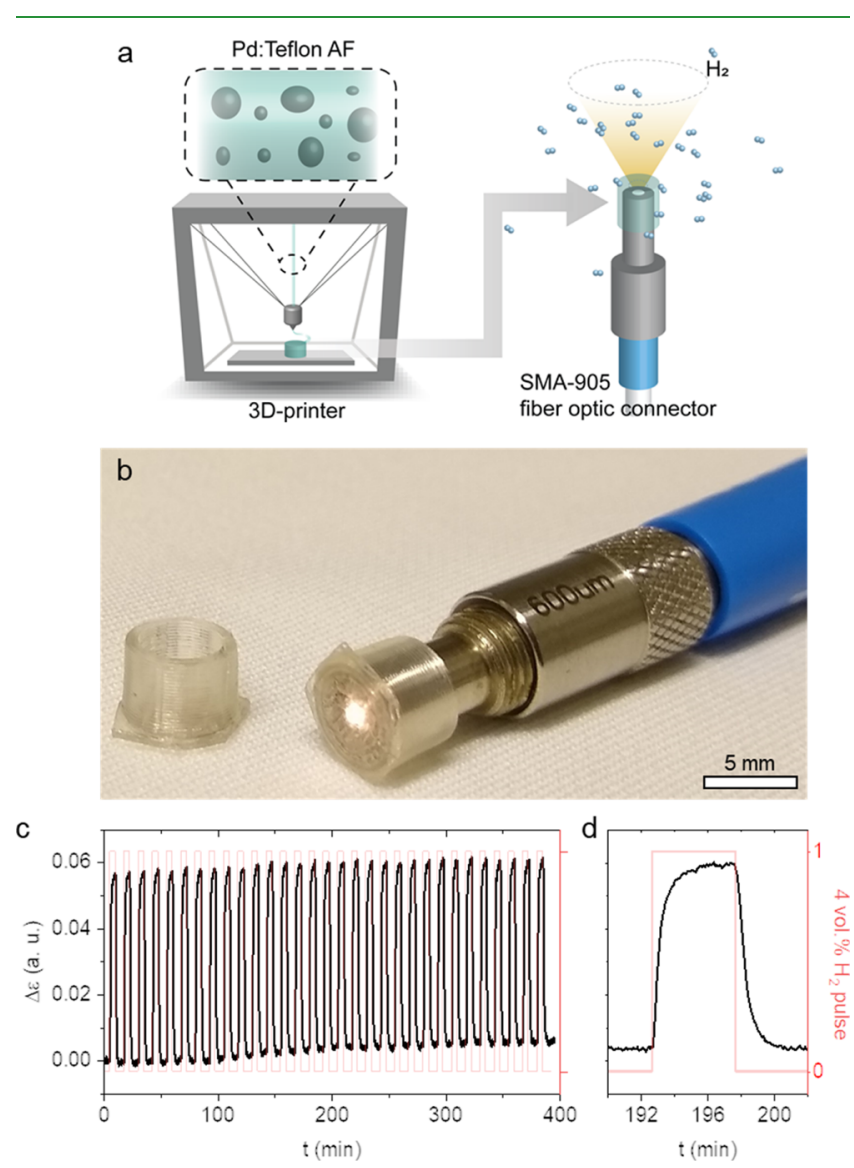

Figure 5. Hydrogen sensing with an optical fiber cap produced by FFF. (a) Fabrication scheme of sensor caps, which are designed to fit by "plug-and-play" onto standard SMA 905 fiber optic connectors; (b) photograph of a sensor cap on an SMA 905 connector, fabricated using a Pd:Teflon AF $\left(3.4 \times 10^{-3}\right.$ vol. \% Pd) filament (note that only the exposed part to hydrogen, i.e., the bottom part, is made of the Teflon nanocomposite); (c) sensor response, $\Delta \varepsilon$, during cyclic exposure to 4 vol. \% hydrogen in synthetic air; and (d) inset: 15 th cycle.

chamber (cf. ref 16 for setup and methodology) in which the sensor can be exposed to pulses of 4 vol. $\% \mathrm{H}_{2}$ in synthetic air in a constant flow configuration to emulate a hydrogen leak. Gratifyingly, the sensor exhibits a stable and reversible response for the investigated cyclic exposure (Figures $5 c$,d and S12). The baseline drift, which appears in Figure 5c, is most likely induced by external factors, such as temperature fluctuation or instability of the light source during the test. The observed apparent longer response and recovery time compared to our previous tests, Figure 4, is the consequence of the gas exchange constant of the chamber, rather than reflecting the intrinsic response/recovery times of the sensor material. We also carried out experiments where we added 500 ppm $\mathrm{CO}$ or $\mathrm{H}_{2} \mathrm{O}$ vapor ( $75 \%$ relative humidity) to the synthetic air mixture and found that the optical response/ recovery time of $\mathrm{Pd}$ :Teflon $\mathrm{AF}$ to $\mathrm{H}_{2}$ slowed down dramatically (not shown). $\mathrm{CO}$ and $\mathrm{H}_{2} \mathrm{O}$ molecules are known to strongly bind to Pd surfaces, thereby blocking $\mathrm{H}_{2}$ sorption. We explain the inability of Teflon AF to protect the $\mathrm{Pd}$ nanoparticles with the relatively large free volume of the polymer, which not only facilitates rapid diffusion of $\mathrm{H}_{2}$ but also allows larger species such as $\mathrm{CO}$ and $\mathrm{H}_{2} \mathrm{O}$ to traverse the matrix.

\section{CONCLUSIONS}

We have prepared nanocomposites of Pd nanoparticles, produced by continuous flow synthesis, and the fluorinated amorphous polymer Teflon AF. Thanks to the high fractional free volume of Teflon AF, the diffusion of $\mathrm{H}_{2}$ through the polymer matrix is sufficiently rapid to not limit the response time of plasmonic sensing based on palladium hydride formation. Melt-pressed plates of Pd:Teflon AF nanocomposites with a thickness of $100 \mu \mathrm{m}$ display a short response time of only $t_{50} \approx 2.5 \mathrm{~s}$ upon exposure to $\mathrm{H}_{2}$ gas (stepwise increase from 0 to $100 \mathrm{mbar}$ in the vacuum background). The sensor exhibits a low LOD down to $30 \mathrm{ppm}$. Finally, we have manufactured sensor caps with FFF, a common 3D printing technique, which can be placed on a standard fiber optic connector and facilitate robust hydrogen sensing. A printed cap displays robust sensing in a long term test of 100 exposures ( $\equiv 18 \mathrm{~h}$ ) to 4 vol. $\% \mathrm{H}_{2}$ in synthetic air. With this demonstration, we argue that melt-processing of $\mathrm{Pd}$ :polymer nanocomposites is a viable route toward the realization of plasmonic plastic $\mathrm{H}_{2}$ sensors.

\section{EXPERIMENTAL SECTION}

Synthesis of PVP-Capped Nanocubes Using Flow Chemistry. All chemicals used were of analytical grade (purity $>99 \%$ ) and used as received from Sigma-Aldrich. All solutions were prepared with MilliQ-water with a resistivity of $18.2 \mathrm{M} \Omega \mathrm{cm}^{-1}$. The automated segmented flow synthesis was performed using a flow system featuring two peristaltic pumps, an air-heated reaction zone and full automation control using the connected computer with integrated software (Vapourtec E-series). Reagents were pumped in high-purity-grade perfluoroalkoxy (PFA) tubes (i.d. $1 \mathrm{~mm}$ ). An aqueous solution of sodium chloropalladate $\left(\mathrm{Na}_{2} \mathrm{PdCl}_{4}\right)(63.3 \mathrm{mM})$ was pumped with a peristaltic pump at a rate of $0.091 \mathrm{~mL} \mathrm{~min}{ }^{-1}$ and was interfaced in a (ethylene tetrafluoroethene, o.d. $1.57 \mathrm{~mm}$ ) T-junction with a mixture of potassium chloride $307.1 \mathrm{mM}$, potassium bromide $5.41 \mathrm{mM}$, polyvinylpyrrolidone $118.2 \mathrm{mM}$, and ascorbic acid $42.7 \mathrm{mM}$, pumped at a rate of $0.242 \mathrm{~mL} \mathrm{~min}{ }^{-1}$. The outlet of the $\mathrm{T}$-junction was connected to a coiled flow reactor $(10 \mathrm{~mL}$; high-purity-grade PFA tubes with i.d. $1 \mathrm{~mm}$ ) which was kept at a constant temperature $(80$ ${ }^{\circ} \mathrm{C}$ ) by hot air (Vapourtec system) and collected in a vial. For medium exchange from water to isopropanol, the Pd suspension was mixed with acetone in the ratio 3:1 (acetone/aqueous suspension of $\mathrm{Pd}$ nanocubes). For instance, $10 \mathrm{~mL}$ of aqueous suspension of $\mathrm{Pd}$ nanocubes was placed in a $50 \mathrm{~mL}$ centrifuge tube and $30 \mathrm{~mL}$ of acetone was added. The mixture was centrifuged at a speed of 3000 $\mathrm{rpm}$ for $5 \mathrm{~min}$. The supernatant was discarded, and $10 \mathrm{~mL}$ of isopropanol was added. The suspension of nanoparticles in 
isopropanol was placed in an ultrasound bath for $5 \mathrm{~min}$ to improve the colloidal stability.

Polymer Nanocomposite Fabrication (Melt Extrusion, Melt Pressing, and 3D Printing). Poly[4,5-difluoro-2,2-bis(trifluoromethyl)-1,3-dioxole-co-tetrafluoroethylene] (AF 1600, here called Teflon AF) with a dioxole content of $65 \mathrm{~mol} \%$ and a density of $1.78 \mathrm{~g} \mathrm{~cm}^{-3}$ was obtained from Chemours; PMMA with a density of $1.2 \mathrm{~g} \mathrm{~cm}^{-3}$, weight-average molecular weight $M_{\mathrm{w}} \approx 75 \mathrm{~kg} \mathrm{~mol}^{-1}$, and a polydispersity index of 2.8 was obtained from Polysciences Inc.; and PVDF with a density of $1.78 \mathrm{~g} \mathrm{~cm}^{-3}$ and a melt flow index of $40 \mathrm{~g} / 10$ $\min \left(230^{\circ} \mathrm{C}, 2.16 \mathrm{~kg}\right)$ was obtained from Solvay Solexis SAS (grade Solef 1006). Different volumes of Pd nanocubes dispersed in isopropanol $(0.3-3 \mathrm{~mL})$ were added to $2 \mathrm{~cm}^{3}$ polymer powder and kept at $50{ }^{\circ} \mathrm{C}$ overnight for drying (removal of isopropanol). The dry mixtures were compounded for $5 \mathrm{~min}$ in an Xplore Microcompounder MC5 at $250{ }^{\circ} \mathrm{C}$. The extrudates were melt-pressed at the same temperatures and $100 \mathrm{kN}$ for $5 \mathrm{~min}$, resulting in a thickness ranging from 50 to $650 \mu \mathrm{m}$.

Fused Filament Fabrication. 3D printing was carried out with a Mass Portal Pharaoh XD printer using a nozzle temperature of 260 ${ }^{\circ} \mathrm{C}$, a build-plate temperature of $60^{\circ} \mathrm{C}$, and a printing speed of 1000 $\mathrm{mm} \min ^{-1}$.

Elemental Analysis. Elemental analysis was carried out at Mikrolab Kolbe, Germany.

Transmission Electron Microscopy. TEM was carried out with a Tecnai T20 microscope with a LaB6 gun, operating at $200 \mathrm{kV}$, or a Titan 80-300 microscope with a field emission gun, operating at 300 $\mathrm{kV}$. The Pd suspension was dropped on a pure carbon 200 mesh copper grid (Ted Pella), while melt-pressed Pd:Teflon AF nanocomposites were microtomed and placed on a Lacey 400 mesh copper grid.

UV-Vis Absorption Spectroscopy. UV-vis absorbance spectra of melt-pressed plates were recorded with a Lambda 1050 spectrophotometer from PerkinElmer.

Scanning SAXS. Scanning SAXS measurements were carried out at the cSAXS (X12SA) beamline of the Swiss Light Source at the Paul Scherrer Institute (PSI) in Villigen PSI, Switzerland. The samples were scanned by a beam with an energy of $11.2 \mathrm{keV}$, which was defined by a fixed-exit double-crystal $\mathrm{Si}(111)$ monochromator and focused to a $28 \times 7.5 \mu \mathrm{m}^{2}$ beam size. A $2 \mathrm{~m}$-long flight tube under vacuum was inserted between the sample and the detector to minimize air scattering and X-ray absorption. The sample-to-detector distance was $2.19 \mathrm{~m}$ calibrated by silver behenate. A Pilatus 2M (1475 $\times 1679$ pixels; pixel size: $172 \times 172 \mu \mathrm{m}^{2}$ ) detector was used to acquire $2 \mathrm{D}$ small-angle scattering patterns. ${ }^{42}$ Simultaneously, the transmitted beam was measured by the fluorescence signal from the steel beamstop. The sample slices (thickness of $200 \mu \mathrm{m}$ ) were placed on a polyimide (Kapton) film (Goodfellow Corp., Cambridge, UK). A raster scan was performed on a chosen sample area of $2 \times 1 \mathrm{~mm}^{2}$ with a step size of $25 \times 10 \mu \mathrm{m}^{2}$ and an exposure time of $0.1 \mathrm{~s}$. Data processing was carried out using the "cSAXS scanning SAXS package" developed by the CXS group, Paul Scherrer Institute, Switzerland. ${ }^{43}$ To determine the size of the Pd particles, form factor fitting was carried out using SASView 4.2.0 on 20 points for each sample. The signal of the Kapton foil and the corresponding pure (nanoparticlefree) polymer matrix was subtracted before the fitting procedure to remove the background signal. The ellipsoid model with the hardsphere structure factor was chosen for the fitting. A polydispersity of 0.1 , determined by trial and error, was applied for determining the size of the ellipsoid.

Thermal Analysis. DSC was carried out with a DSC2 from Mettler Toledo under nitrogen $\left(100 \mathrm{~mL} \mathrm{~min}^{-1}\right)$, between 25 and 300 ${ }^{\circ} \mathrm{C}$, at a scan rate of $10^{\circ} \mathrm{C} \mathrm{min}^{-1}$. The sample weight was 5-10 mg.

Hydrogen Permeability. The hydrogen transport properties were determined by means of direct permeation experiments using a manometric technique (ASTM Standard norm D 1434), and the penetrant flux through the samples was obtained from the pressure increase in a calibrated closed volume, starting from high vacuum conditions. ${ }^{44}$ A capacitive gauge (Edwards Barocel, 0-100 mbar range, sensitivity 0.01 mbar) measured the gas pressure in the downstream chamber, while the upstream pressure was kept constant at approximately $1.2-1.5$ bar (Druck, $0-10 \mathrm{bar}$ ). Tests were carried out under isothermal conditions at $30^{\circ} \mathrm{C}$ in a thermostatic chamber (PID, $\pm 0.1 \mathrm{~K})$. Samples were first placed in the sample holder (Millipore, permeation area $2.2 \mathrm{~cm}^{2}$ ) set across the two closed volumes and treated overnight under vacuum to remove all possible adsorbed species. The tests were performed out by connecting a highpressure chamber with the specimen and were stopped only after steady-state conditions are achieved, observed as a linear increase in downstream pressure over time. Each test was repeated at least twice to ensure the reproducibility of the data obtained. The gas permeability $P$ was calculated as follows

$$
P=J_{\text {ss }} \frac{l}{\Delta p}=\left(\frac{d p_{\mathrm{d}}}{t}\right)_{\vec{t}+\infty} \frac{V_{\mathrm{d}}}{R T} \frac{l}{A \Delta p}
$$

where $J_{s s}$ is the penetrant molar flux per unit area (at the steady state), $\Delta p$ is the pressure difference, $p_{\mathrm{d}}$ is the downstream pressure, $V_{\mathrm{d}}$ is the calibrated volume of the downstream side, and $A$ is the membrane area. Diffusion kinetics were evaluated by the time-lag method, which represents the process characteristic time, and the value of $\theta_{\mathrm{L}}$ was determined as the intercept on the time axis of the linear $p_{\mathrm{d}}$ versus $t$ behavior at long times, once steady-state conditions were attained; the diffusivity $D$ was thus calculated as ${ }^{45}$

$$
D=\frac{l^{2}}{6 \theta_{\mathrm{L}}}
$$

Hydrogen Sensing Measurements. $\mathrm{H}_{2}$ sensing was carried out with two platforms: (i) a home-built vacuum chamber, for the experiment in Figure 4, and (ii) a mini flow reactor, for the experiment in Figure 5. The setups are described in detail elsewhere. ${ }^{16}$ Experiment (i) was carried out at $30^{\circ} \mathrm{C}$ using a feedback-loop heating system comprising a heating coil, a power supply, and a PID thermocontroller (Eurotherm 3216). Experiment (ii) was performed at $21{ }^{\circ} \mathrm{C}$ (the ambient lab temperature). Both experiments were executed in the transmission mode, where a polychromatic halogen light (Avantes AvaLight-Hal Mini) was directed toward the sensor and the transmitted light was monitored using a visible light-range spectrophotometer (Avantes SensLine AvaSpec-2048XL). In experiment (i), the optical response of the sensor was monitored during the quasi-static, (de)increasing hydrogen pressure controlled using leak valves (for the isotherm measurement), and during a sudden hydrogen pressure change using pneumatic valves (for the kinetic measurement). In the case of experiment (ii), the optical response was monitored during cycles of exposure to 4 vol. $\% \mathrm{H}_{2}$ (diluted in synthetic air and total flow $=200 \mathrm{ml} \mathrm{min}{ }^{-1}$ ). The gas flow and mixture were controlled by mass flow controllers (Bronkhorst Low $\Delta \mathrm{P})$.

\section{ASSOCIATED CONTENT}

\section{Supporting Information}

The Supporting Information is available free of charge at https://pubs.acs.org/doi/10.1021/acsami.1c01968.

TEM images of the Pd single crystal and Pd nanoparticles in Teflon AF, UV-vis absorbance spectra of Pd:Teflon AF plates; elemental analysis for obtaining the Pd concentration in Pd:Teflon AF plates; SAXS of Pd:Teflon AF nanocomposites; DSC melting thermograms for PMMA, Teflon AF, and PVDF; isotherms of different Pd loading Pd:Teflon plates; $\Delta \varepsilon, t_{50}$, and optical contrast of Pd:Teflon AF as functions of thickness and Pd content upon hydrogen absorption and desorption; Pd:Teflon sensor response at low hydrogen pressures and the limit of detection and $t_{50}$ during hydrogen cycling exposure for the $3 \mathrm{D}$-printed sensor (PDF) 


\section{AUTHOR INFORMATION}

\section{Corresponding Authors}

Kasper Moth-Poulsen - Department of Chemistry and Chemical Engineering, Chalmers University of Technology, Göteborg 412 96, Sweden; 이이.org/0000-0003-40184927; Email: moth-poulsen@chalmers.se

Christoph Langhammer - Department of Physics, Chalmers University of Technology, Göteborg 412 96, Sweden; 다이.org/0000-0003-2180-1379; Email: clangham@ chalmers.se

Christian Müller - Department of Chemistry and Chemical Engineering, Chalmers University of Technology, Göteborg 412 96, Sweden; (i) orcid.org/0000-0001-7859-7909; Email: christian.muller@chalmers.se

\section{Authors}

Ida Östergren - Department of Chemistry and Chemical Engineering, Chalmers University of Technology, Göteborg 412 96, Sweden; 이이이.org/0000-0002-2431-0746

Amir Masoud Pourrahimi - Department of Chemistry and Chemical Engineering, Chalmers University of Technology, Göteborg 412 96, Sweden; 이이.org/0000-0001-58670531

Iwan Darmadi - Department of Physics, Chalmers University of Technology, Göteborg 412 96, Sweden; - orcid.org/ 0000-0002-5921-9336

Robson da Silva - Department of Chemistry and Chemical Engineering, Chalmers University of Technology, Göteborg 412 96, Sweden

Alicja Stolas - Department of Chemistry and Chemical Engineering, Chalmers University of Technology, Göteborg 412 96, Sweden; (1) orcid.org/0000-0002-6736-9553

Sarah Lerch - Department of Chemistry and Chemical Engineering, Chalmers University of Technology, Göteborg 412 96, Sweden; 이이. orcid.org/0000-0001-5968-8178

Barbara Berke - Department of Physics, Chalmers University of Technology, Göteborg 412 96, Sweden; 이이.org/ 0000-0002-3105-2036

Manuel Guizar-Sicairos - Paul Scherrer Institut, Villigen PSI 5232, Switzerland

Marianne Liebi - Department of Physics, Chalmers University of Technology, Göteborg 412 96, Sweden; (1) orcid.org/ 0000-0002-5403-0593

Giacomo Foli - Institute of Organic Synthesis and Photoreactivity, National Research Council, Bologna 40129, Italy

Vincenzo Palermo - Institute of Organic Synthesis and Photoreactivity, National Research Council, Bologna 40129, Italy; Department of Industrial and Materials Science, Chalmers University of Technology, Göteborg 412 96, Sweden

Matteo Minelli - Department of Civil, Chemical, Environmental and Materials Engineering, Alma Mater Studiorum-University of Bologna, Bologna 40131, Italy; (1) orcid.org/0000-0003-4662-1526

Complete contact information is available at: https://pubs.acs.org/10.1021/acsami.1c01968

\section{Author Contributions}

II.Ö., A.M.P., and I.D. contributed equally.

\section{Notes}

The authors declare no competing financial interest.

\section{ACKNOWLEDGMENTS}

The authors acknowledge financial support from the Swedish Foundation for Strategic Research projects RMA15-0052 and FFL15-0147 and the Knut and Alice Wallenberg Foundation project 2016.0210. The authors also acknowledge the Centre for Cellular Imaging at the University of Gothenburg and the National Microscopy Infrastructure, NMI (VR-RFI 201600968), for providing assistance with microtome sectioning. Finally, the authors thank the Swiss Light Source at the Paul Scherrer Institute, Switzerland, for provision of synchrotron radiation beamtime at the cSAXS beamline. Part of this work was carried out at the Chalmers Materials Analysis Laboratory (CMAL).

\section{REFERENCES}

(1) Qian, J. X.; Chen, T. W.; Enakonda, L. R.; Liu, D. B.; Mignani, G.; Basset, J.-M.; Zhou, L. Methane Decomposition to Produce COxfree Hydrogen and Nano-Carbon over Metal Catalysts: A Review. Int. J. Hydrogen Energy 2020, 45, 7981-8001.

(2) Cao, L.; Yu, I. K. M.; Xiong, X.; Tsang, D. C. W.; Zhang, S.; Clark, J. H.; Hu, C.; Ng, Y. H.; Shang, J.; Ok, Y. S. Biorenewable Hydrogen Production through Biomass Gasification: A Review and Future Prospects. Environ. Res. 2020, 186, 109547.

(3) Safari, F.; Dincer, I. A Review and Comparative Evaluation of Thermochemical Water Splitting Cycles for Hydrogen Production. Energy Convers. Manage. 2020, 205, 112182.

(4) Pourrahimi, A. M.; Andersson, R. L.; Tjus, K.; Ström, V.; Björk, A.; Olsson, R. T. Making an Ultralow Platinum Content Bimetallic Catalyst on Carbon Fibres for Electro-Oxidation of Ammonia in Wastewater. Sustainable Energy Fuels 2019, 3, 2111-2124.

(5) De Vrieze, J.; Verbeeck, K.; Pikaar, I.; Boere, J.; Van Wijk, A.; Rabaey, K.; Verstraete, W. The Hydrogen Gas Bio-Based Economy and the Production of Renewable Building Block Chemicals, Food and Energy. New Biotechnol. 2020, 55, 12-18.

(6) Pareek, A.; Dom, R.; Gupta, J.; Chandran, J.; Adepu, V.; Borse, P. H. Insights into Renewable Hydrogen Energy: Recent Advances and Prospects. Mater. Sci. Energy Technol. 2020, 3, 319-327.

(7) Dutta, S. A Review on Production, Storage of Hydrogen and its Utilization as an Energy Resource. J. Ind. Eng. Chem. 2014, 20, 11481156.

(8) Nugroho, F. A. A.; Darmadi, I.; Cusinato, L.; Susarrey-Arce, A.; Schreuders, H.; Bannenberg, L. J.; da Silva Fanta, A. B.; Kadkhodazadeh, S.; Wagner, J. B.; Antosiewicz, T. J.; Hellman, A.; Zhdanov, V. P.; Dam, B.; Langhammer, C. Metal-Polymer Hybrid Nanomaterials for Plasmonic Ultrafast Hydrogen Detection. Nat. Mater. 2019, 18, 489-495.

(9) Wadell, C.; Syrenova, S.; Langhammer, C. Plasmonic Hydrogen Sensing with Nanostructured Metal Hydrides. ACS Nano 2014, 8, 11925-11940.

(10) Darmadi, I.; Nugroho, F. A. A.; Langhammer, C. HighPerformance Nanostructured Palladium-Based Hydrogen SensorsCurrent Limitations and Strategies for Their Mitigation. ACS Sens. 2020, 5, 3306-3327.

(11) Darmadi, I.; Nugroho, F. A. A.; Kadkhodazadeh, S.; Wagner, J. B.; Langhammer, C. Rationally Designed PdAuCu Ternary Alloy Nanoparticles for Intrinsically Deactivation-Resistant Ultrafast Plasmonic Hydrogen Sensing. ACS Sens. 2019, 4, 1424-1432.

(12) Penner, R. M. A Nose for Hydrogen Gas: Fast, Sensitive H2 Sensors Using Electrodeposited Nanomaterials. Acc. Chem. Res. 2017, 50, 1902-1910.

(13) Hong, J.; Lee, S.; Seo, J.; Pyo, S.; Kim, J.; Lee, T. A Highly Sensitive Hydrogen Sensor with Gas Selectivity Using a PMMA Membrane-Coated Pd Nanoparticle/Single-Layer Graphene Hybrid. ACS Appl. Mater. Interfaces 2015, 7, 3554-3561.

(14) Chen, M.; Mao, P.; Qin, Y.; Wang, J.; Xie, B.; Wang, X.; Han, D.; Wang, G.-h.; Song, F.; Han, M.; Liu, J.-M.; Wang, G. Response Characteristics of Hydrogen Sensors Based on PMMA-Membrane- 
Coated Palladium Nanoparticle Films. ACS Appl. Mater. Interfaces 2017, 9, 27193-27201.

(15) Xiao, M.; Liang, S.; Han, J.; Zhong, D.; Liu, J.; Zhang, Z.; Peng, L. Batch Fabrication of Ultrasensitive Carbon Nanotube Hydrogen Sensors with Sub-ppm Detection Limit. ACS Sens. 2018, 3, 749-756. (16) Darmadi, I.; Stolaś, A.; Östergren, I.; Berke, B.; Nugroho, F. A. A.; Minelli, M.; Lerch, S.; Tanyeli, I.; Lund, A.; Andersson, O.; Zhdanov, V. P.; Liebi, M.; Moth-Poulsen, K.; Müller, C.; Langhammer, C. Bulk-Processed Pd Nanocube-Poly(methyl methacrylate) Nanocomposites as Plasmonic Plastics for Hydrogen Sensing. ACS Appl. Nano Mater. 2020, 3, 8438-8445.

(17) Herkert, E.; Sterl, F.; Strohfeldt, N.; Walter, R.; Giessen, H. Low-Cost Hydrogen Sensor in the ppm Range with Purely Optical Readout. ACS Sens. 2020, 5, 978-983.

(18) Sun, C.; Lu, P.; Wright, R.; Ohodnicki, P. Low-Cost Fiber Optic Sensor Array for Simultaneous Detection of Hydrogen and Temperature. Fiber Optic Sensors and Applications XV; Proceedings of SPIE: Bellingham, WA, 2018; Vol. 10654.

(19) Clémenson, S.; Espuche, E.; David, L.; Léonard, D. Nanocomposite Membranes of Polyetherimide Nanostructured with Palladium Particles: Processing Route, Morphology and Functional Properties. J. Membr. Sci. 2010, 361, 167-175.

(20) Wang, W.; Zhang, S.; Srisombat, L.-o.; Lee, T. R.; Advincula, R. C. Gold-Nanoparticle- and Gold-Nanoshell-Induced Polymorphism in Poly(vinylidene fluoride). Macromol. Mater. Eng. 2011, 296, 178184.

(21) Pandit, P.; Schwartzkopf, M.; Rothkirch, A.; Roth, S. V.; Bernstorff, S.; Gupta, A. Structure-Function Correlations in Sputter Deposited Gold/Fluorocarbon Multilayers for Tuning Optical Response. Nanomaterials 2019, 9, 1249.

(22) Schwartzkopf, M.; Wöhnert, S.-J.; Waclawek, V.; Carstens, N.; Rothkirch, A.; Rubeck, J.; Gensch, M.; Drewes, J.; Polonskyi, O.; Strunskus, T.; Hinz, A. M.; Schaper, S. J.; Körstgens, V.; MüllerBuschbaum, P.; Faupel, F.; Roth, S. V. Real-time Insight into Nanostructure Evolution during the Rapid Formation of Ultra-Thin Gold Layers on Polymers. Nanoscale Horiz. 2021, 6, 132-138.

(23) Delmelle, R.; Ngene, P.; Dam, B.; Bleiner, D.; Borgschulte, A. Promotion of Hydrogen Desorption from Palladium Surfaces by Fluoropolymer Coating. ChemCatChem 2016, 8, 1646-1650.

(24) Pourrahimi, A. M.; Hoang, T. A.; Liu, D.; Pallon, L. K. H.; Gubanski, S.; Olsson, R. T.; Gedde, U. W.; Hedenqvist, M. S. Highly Efficient Interfaces in Nanocomposites Based on Polyethylene and $\mathrm{ZnO}$ Nano/Hierarchical Particles: A Novel Approach toward Ultralow Electrical Conductivity Insulations. Adv. Mater. 2016, 28 , $8651-8657$.

(25) Minelli, M.; Sarti, G. C. Elementary Prediction of Gas Permeability in Glassy Polymers. J. Membr. Sci. 2017, 521, 73-83.

(26) Minelli, M.; Sarti, G. C. Gas Permeability in Glassy Polymers: A Thermodynamic Approach. Fluid Phase Equilib. 2016, 424, 44-51.

(27) Pekkari, A.; Say, Z.; Susarrey-Arce, A.; Langhammer, C.; Härelind, H.; Sebastian, V.; Moth-Poulsen, K. Continuous Microfluidic Synthesis of Pd Nanocubes and PdPt Core-Shell Nanoparticles and Their Catalysis of NO2 Reduction. ACS Appl. Mater. Interfaces 2019, 11, 36196-36204.

(28) Ansaloni, L.; Deng, L. 7-Advances in Polymer-Inorganic Hybrids as Membrane Materials. In Recent Developments in Polymer Macro, Micro and Nano Blends; Visakh, P. M., Markovic, G., Pasquini, D., Eds.; Woodhead Publishing, 2017; pp 163-206.

(29) Langhammer, C.; Yuan, Z.; Zorić, I.; Kasemo, B. Plasmonic Properties of Supported Pt and Pd Nanostructures. Nano Lett. 2006, 6, 833-838.

(30) Pradeep, T. Verification of the Beer-Lambert Law using Gold Nanoparticles. Textbook of Nanoscience and Nanotechnology; McGrawHill Education: New York, 2012.

(31) Emami, N.; Sjödahl, M.; Söderholm, K.-J. M. How Filler Properties, Filler Fraction, Sample Thickness and Light Source affect Light Attenuation in Particulate Filled Resin Composites. Dent. Mater. 2005, 21, 721-730.
(32) Giacinti Baschetti, M.; Minelli, M. Test Methods for the Characterization of Gas and Vapor Permeability in Polymers for Food Packaging Application: A Review. Polym. Test. 2020, 89, 106606.

(33) Ferrari, M. C.; Galizia, M.; De Angelis, M. G.; Sarti, G. C. Gas and Vapor Transport in Mixed Matrix Membranes Based on Amorphous Teflon AF1600 and AF2400 and Fumed Silica. Ind. Eng. Chem. Res. 2010, 49, 11920-11935.

(34) Alekseeva, S.; Fanta, A. B. d. S.; Iandolo, B.; Antosiewicz, T. J.; Nugroho, F. A. A.; Wagner, J. B.; Burrows, A.; Zhdanov, V. P.; Langhammer, C. Grain Boundary Mediated Hydriding Phase Transformations in Individual Polycrystalline Metal Nanoparticles. Nat. Commun. 2017, 8, 1084.

(35) Syrenova, S.; Wadell, C.; Nugroho, F. A. A.; Gschneidtner, T. A.; Diaz Fernandez, Y. A.; Nalin, G.; Switlik, D.; Westerlund, F.; Antosiewicz, T. J.; Zhdanov, V. P.; Moth-Poulsen, K.; Langhammer, C. Hydride Formation Thermodynamics and Hysteresis in Individual Pd Nanocrystals with Different Size and Shape. Nat. Mater. 2015, 14, 1236-1244.

(36) Fisser, M.; Badcock, R. A.; Teal, P. D.; Hunze, A. Optimizing the Sensitivity of Palladium based Hydrogen Sensors. Sens. Actuators, B 2018, 259, 10-19.

(37) Wadell, C.; Nugroho, F. A. A.; Lidström, E.; Iandolo, B.; Wagner, J. B.; Langhammer, C. Hysteresis-Free Nanoplasmonic PdAu Alloy Hydrogen Sensors. Nano Lett. 2015, 15, 3563-3570.

(38) Johnson, N. J. J.; Lam, B.; MacLeod, B. P.; Sherbo, R. S.; Moreno-Gonzalez, M.; Fork, D. K.; Berlinguette, C. P. Facets and Vertices Regulate Hydrogen Uptake and Release in Palladium Nanocrystals. Nat. Mater. 2019, 18, 454-458.

(39) Stolaś, A.; Darmadi, I.; Nugroho, F. A. A.; Moth-Poulsen, K.; Langhammer, C. Impact of Surfactants and Stabilizers on Palladium Nanoparticle-Hydrogen Interaction Kinetics: Implications for Hydrogen Sensors. ACS Appl. Nano Mater. 2020, 3, 2647-2653.

(40) Palm, K. J.; Murray, J. B.; Narayan, T. C.; Munday, J. N. Dynamic Optical Properties of Metal Hydrides. ACS Photonics 2018, 5, 4677-4686.

(41) Nugroho, F. A. A.; Darmadi, I.; Zhdanov, V. P.; Langhammer, C. Universal Scaling and Design Rules of Hydrogen-Induced Optical Properties in Pd and Pd-Alloy Nanoparticles. ACS Nano 2018, 12, 9903-9912.

(42) Kraft, P.; Bergamaschi, A.; Broennimann, C.; Dinapoli, R.; Eikenberry, E. F.; Henrich, B.; Johnson, I.; Mozzanica, A.; Schlepütz, C. M.; Willmott, P. R.; Schmitt, B. Performance of Single-PhotonCounting PILATUS Detector Modules. J. Synchrotron Radiat. 2009, 16, 368-375.

(43) Bunk, O.; Bech, M.; Jensen, T. H.; Feidenhans'l, R.; Binderup, T.; Menzel, A.; Pfeiffer, F. Multimodal X-Ray Scatter Imaging. New J. Phys. 2009, 11, 123016.

(44) Minelli, M.; De Angelis, M. G.; Doghieri, F.; Marini, M.; Toselli, M.; Pilati, F. Oxygen Permeability of Novel OrganicInorganic Coatings: I. Effects of Organic-Inorganic Ratio and Molecular Weight of the Organic Component. Eur. Polym. J. 2008, 44, 2581-2588.

(45) Crank, J. The Mathematics of Diffusion, 2nd ed.; Clarendon Press: Oxford, 1979. 